\title{
Assessing Infrastructure System of Systems Integrity
}

\author{
R. Peculis a , F. Shirvani ${ }^{\text {a }}$ and $\underline{\text { P. Perez }}^{\text {a }}$ \\ ${ }^{a}$ SMART Infrastructure Facility, Faculty of Engineering and Information Sciences \\ University of Wollongong \\ Email: ricardo_peculis@uow.edu.au
}

\begin{abstract}
Infrastructure refers to the fundamental facilities and systems serving a country, city, or area, including the services and facilities necessary for its economy to function. Infrastructure systems typically consist of interrelated constituent systems forming what is known as system of systems (SoS). Infrastructure systems present numerous challenges throughout their lifecycles. This paper addresses one of these challenges that is presented during operation, when managers need to report 'how well' the system is performing and finding ways to address the consequences of unexpected events that often degrade the intended performance. This state of system 'wellbeing' will be referred as system integrity (SI).
\end{abstract}

When applied to infrastructure systems this paper proposes a model suggesting that system integrity is a combination of operational performance, safety and resilience which become the set of criteria to assess SI. Each of these three factors is assessed by considering their specific 'key performance indicators' (KPI): Operational KPIs (KO), Safety KPIs (KS) and Resilience KPIs (KR). KOs could include KPIs for quality of service, reliability, availability, maintainability and cost; KSs could include KPIs for number and severity of accidents; and KRs could include KPIs for level of disruption and time for recovery to acceptable levels.

In accordance with the proposed model system integrity can be defined as the "state of a system where it is performing its intended functions safely without being degraded or impaired by changes or disruptions in its internal or external environments". When the system achieves the state of perfect condition its system integrity is $100 \%$ or 1.0 . Infrastructure systems may operate at lesser levels of system integrity (SI) and it is important to assess and monitor SI to make sure the system is operating within acceptable levels and to envisage ways to improve SI in the event of unexpected situations.

The proposed model based on the on-going operational performance, safety and resilience of the each constituent system in the SoS is then developed into a method that applies the Analytic Hierarchy Process (AHP) (Saaty 1994) to create a quantitative assessment derived from qualitative and quantitative information. The method assumes that there is a set of KPIs for each of the agreed assessment criterion for operational performance, safety and resilience which were defined, agreed and can be individually assessed. The method uses qualitative experience-based information to weight the KPIs for each of the three criteria relatively to each other using AHP to obtain the overall assessment for operational performance, safety and resilience for each individual constituent system. These three criteria are also compared and weighted using the same approach to determine their level of contribution to SI which is then calculated using the actual value measured or estimated for each KPI. The method is then expanded to calculate the SI for SoS by applying the concept of 'supermatrix' proposed by AHP to address systems with feedback loops where individual components and assessment criteria influence each other. The SoS SI method is then applied into a hypothetical urban transport system for illustration purposes.

Keywords: Infrastructure, system of systems, system integrity, urban transport 
Peculis and Shirvani, Assessing Infrastructure System of Systems Integrity

\section{INTRODUCTION}

Infrastructure systems typically characterises technical structures such as transport systems which can be defined as the physical and operational components of interrelated systems forming what is now known as system of systems. Although there is no single definition for system of systems (SoS), there is a consensus that SoS exhibit 'emergent behaviours' that result from the interaction of constituent systems that are operated and managed independently (Nielsen, Larsen et al. 2015). Another definition for SoS is provided in (Mayk and Madni 2006) as "a collection of systems that were originally designed as stand-alone systems for specific and different purposes but that have been brought together within the SoS umbrella to create a new capability needed for a particular mission". Man-made SoS, like infrastructure systems, are designed to exhibit desirable behaviours which are the objectives for the system in the first place.

Infrastructure systems present numerous challenges throughout their lifecycles, from concept definition, planning, design and construction through operation and final disposal. One of these challenges is presented during operation when managers need to report 'how well' the system is performing and will continue performing in the future. It is also under the responsibility of infrastructure managers to find ways to address the consequences of unexpected events that often degrade the intended performance. This state of 'system wellbeing' will be referred as system integrity (SI).

There is no standard or agreed definition for system integrity in the context of infrastructure systems in the published literature. The NASA systems Engineering handbook (Kapurch 2010) defines the system integrity as "the efficient composition of components/subsystems into a whole that offers the required functionality and achieves specific goals". According to (Neches and Madni 2013) complex systems of today have to satisfy a number of requirements such as affordability, reliability, adaptability, security, and resilience, increasing even further the difficulty in assessing system integrity. SI is integral to the overall system development life cycle. A simplified SI process, based on the traditional waterfall model that has been used for decades, includes: validation testing, which focuses on whether or not the system performs the functions that are needed, and verification testing, which assures compliance with formally defined requirements (Madni and Sievers 2014).

Within the context of infrastructure systems this paper suggests that system integrity is a combination of three attributes of operational performance, safety and resilience that become the criteria for assessing system integrity. In this context SI can be defined as the "state of a system where it is performing its intended functions safely without being degraded or impaired by changes or disruptions in its internal or external environments", adapted from the (Business Dictionary 2017). Each criterion of operational performance, safety and resilience needs to be assessed in accordance with a set of agreed 'key performance indicators' (KPIs) (Figure 1). Operational performance is an indication of how well the system is when compared against 'key operational

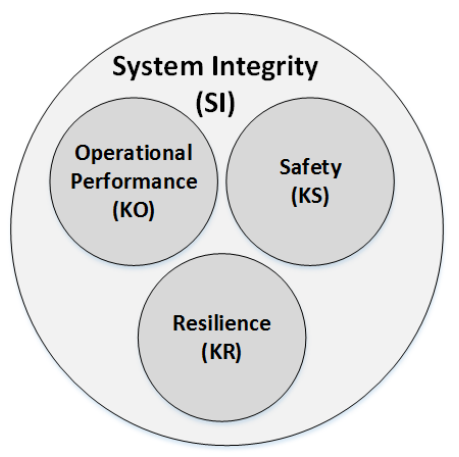

Figure 1. SI Model for a single system performance indicators' (KO) for level of service, reliability, availability and maintainability. Safety is the ability of the system to perform safely, i.e. without the risk of loss of life or injuring people and damaging properties and assets, and is assessed against 'key safety indicators' (KS). Resilience can be assessed against 'key resilience indicators' (KR) reporting the capacity of the system to recover from disruptions and continuing operating in adverse and sometimes unpredictable conditions (Pyster, Olwell et al. 2017). When the system achieves its perfect condition its system integrity is $100 \%$ or 1.0 . Infrastructure systems may operate at lesser levels of system integrity (SI) and it is important to assess and monitor SI to make sure the system is operating within acceptable levels and to envisage ways to improve SI in the event of unexpected situations.

This paper proposes a model to assess SI of SoS based on the on-going operational performance, safety and resilience of each constituent system. A method applying the Analytic Hierarchy Process (AHP) (Saaty 1994) is then presented to create a quantitative assessment of SI derived from qualitative and quantitative information.

The paper is organized as follows. Section 2 discusses SI for one single system and proposes a method for assessing SI of infrastructure systems using AHP. Section 3 introduces SI for SoS, proposes a method to assess SI of SoS and presents a hypothetical example applying the proposed method. Section 4 concludes the paper and suggests future work. 
Peculis and Shirvani, Assessing Infrastructure System of Systems Integrity

\section{ASSESSING SYSTEM INTEGRITY}

From the context and definition presented in the introduction SI is a combination of operational performance, safety and resilience. System integrity is the result of assessing how well the system achieves the three criteria of operational performance, safety and resilience. The proposed model assumes that these three criteria are independent for a single system, as shown in Figure 1. Resilience is assumed to be achieved by design and is embedded into system and through redundancy, backup and alternative modes of operation. Components that provide resilience are activated only when needed to compensate other components that may not be fully operational. The following six steps method based on the proposed model, summarised in Table 1, is then used to assess the system integrity of a single system.

Table 1. Six step method for assessing the SI of a single system

\begin{tabular}{|l|l|}
\hline Step 1 & $\begin{array}{l}\text { Define the 'key performance indicators' (KPI) used to asses each of the three criteria: KO for operational performance, } \\
\text { KS for safety and KR for resilience. Each criterion potentially has many KPIs and each KPI should have a method to } \\
\text { be measured or assessed. }\end{array}$ \\
\hline Step 2 & $\begin{array}{l}\text { For each criterion compare pairwise its KPIs to obtain their relative importance using AHP Priority Matrix. This will } \\
\text { provide the relative weight of each KPI for a given criterion. }\end{array}$ \\
\hline Step 3 & $\begin{array}{l}\text { Using the methods defined in Step 1 assess the actual value for each KPI in a scale from 0.0 to 1.0, the latter means the } \\
\text { KPI has been fully achieved. }\end{array}$ \\
\hline Step 4 & $\begin{array}{l}\text { Using the weights obtained in Step } 2 \text { and the actual value for each KPI the overall Operational Performance, Safety } \\
\text { and Resilience of the system are calculated. }\end{array}$ \\
\hline Step 5 & $\begin{array}{l}\text { Using AHP Priority Matrix compare pairwise the three criteria to obtain the relative weight of each criteria in the } \\
\text { context of system integrity. }\end{array}$ \\
\hline Step 6 & $\begin{array}{l}\text { System integrity is calculated using the actual values for Operational Performance, Safety and Resilience obtained in } \\
\text { Step } 4 \text { and the weights from Step 5. }\end{array}$ \\
\hline
\end{tabular}

Step 1

The KPIs for operational performance, safety and resilience must be well defined and understood and there are methods to measure or to assess how well the system is achieving each KPI. The definition of KPIs and their methods of assessment are out of scope of this paper. It is assumed that the engineers, managers and other people in the organization are capable of defining and assessing their pertinent KPIs.

\section{Step 2}

The Analytic Hierarchy Process (AHP) is a framework of multi-valued logic based on the innate human ability to use information and experience to construct ratio scales through paired comparison (Saaty 2000). The object of the analysis is arranged in a hierarchic network structure that breaks down the whole into its smaller parts thus allowing paired comparison. Paired comparison is done using 'The Fundamental Scale' of nine levels 1-9, shown in Table 2 (adapted from (Saaty 1994) Table 3.1 and (DiMario, Boardman et al. 2009) Table II).

Table 2. The Fundamental Scale of AHP

\begin{tabular}{|c|l|l|}
\hline Intensity of Importance & Definition & Explanation \\
\hline 1 & Equal importance & The two components contribute equally to the objective \\
\hline 3 & Moderate importance & Experience and judgement slightly favour one component over the other \\
\hline 5 & Strong importance & Experience and judgement strongly favour one component over the other \\
\hline 7 & Very strong importance & $\begin{array}{l}\text { One component is favoured very strongly over another; its dominance } \\
\text { demonstrated in practice }\end{array}$ \\
\hline 9 & Extreme importance & $\begin{array}{l}\text { The evidence favouring one component over another is of the highest possible } \\
\text { order of affirmation }\end{array}$ \\
\hline $2,4,6,8$ & $\begin{array}{l}\text { For comparisons between } \\
\text { the above values }\end{array}$ & Interpolation of a compromised judgement \\
\hline
\end{tabular}

The objective of the AHP is to compare all components in the system of interest to determine the weight of importance or contribution of each component to the whole. Although AHP has formal mathematical foundation it is simple to use and algebraic calculations are easily performed with the aid of mathematical software tools. AHP shows that if the system of interest has ' $n$ ' components, the pair comparison constitutes an $n \times n$ square matrix named Priority Matrix.

Considering that the operational performance criterion is described by ' $\mathrm{n}$ ' KOs which a team of experienced people should be able to discuss and hopefully agree with how each $\mathrm{KO}$ is more or less important to the overall performance. The assessment is done by comparing each $\mathrm{KO}$ with the other ' $\mathrm{n}-1$ ' KOs and placing 
the comparison in a square matrix, as shown in Table 3. The diagonal of the matrix is ' 1 ' as it corresponds to the comparison of a $\mathrm{KO}$ with itself. The components $\mathrm{C}_{\mathrm{i}, \mathrm{j}}$ above the diagonal are numbers from ' 1 to 9' chosen from the 'the fundamental scale of AHP' while the components below the diagonal are the inverse of the components above because they correspond to the inverse comparison, i.e. 'how is $K O_{i}$ more important than $K O_{j}$ ?' and 'how is $K \mathrm{O}_{j}$ more important than $\mathrm{KO}_{i}$ ?'

The relative weight or priority of each KPI is the normalised principal eigenvector, obtained from the maximum eigenvalue, of the Performance Priority Matrix (Saaty 1994) and can be calculated using mathematical tools such as $M A T L A B$ or other similar tools. The Performance Vector for the component ' $c$ ' (OPVc), equation (1), corresponds to the weight (W) of importance of each of the 'n' KOs to the overall performance. OPVc needs to be normalised so that the some of its components is equal to ' 1.0 ', as shown by equation (2). The same process is repeated for the other two criteria of safety and resilience to obtain the relative weight of their respective KPIs.

\section{Steps 3 and 4}

In practice the components contributing to the system performance may not be performing at their nominal capacity reflecting the 'actual performance' (AP) that could be between $0 \%$ and $100 \%$. The level of 'acceptable performance', 'degraded performance' or 'not operational' can be defined by AP ranges and thresholds. AP is calculated or estimated in accordance with predefined and agreed methods. The set of values of 'how well KPIs are achieved' form the Actual Operational Performance Vector (AOPVc) and the product of OPVc and AOPVc transposed is the operational performance $(\mathrm{OPc})$ of that system component, as shown by equation (3).

Table 3. Operational Performance Priority Matrix for a single component

\begin{tabular}{|c|c|c|c|c|c|}
\hline & $\mathrm{KO}_{1}$ & $\mathrm{KO}_{2}$ & $\ldots$ & $K \mathbf{O}_{\mathbf{n}-1}$ & $\mathrm{KO}_{\mathbf{n}}$ \\
\hline $\mathrm{KO}_{1}$ & 1 & $\mathbf{C}_{1,2}$ & $\ldots$ & $\mathrm{C}_{1, \mathrm{n}-1}$ & $\mathbf{C}_{1, \mathbf{n}}$ \\
\hline $\mathrm{KO}_{2}$ & $1 / C_{1,2}$ & 1 & $\ldots$ & $\mathrm{C}_{2, \mathrm{n}-1}$ & $\mathrm{C}_{2, \mathrm{n}}$ \\
\hline$\ldots$ & $\ldots$ & $\ldots$ &.. & $\ldots$ & $\ldots$ \\
\hline$K O_{n-1}$ & $1 / C_{1, n-1}$ & $1 / C_{2, n-1}$ & $\ldots$ & 1 & $C_{n-1, n}$ \\
\hline $\mathrm{KO}_{\mathbf{n}}$ & $1 / C_{1, n}$ & $1 / C_{2, n}$ & $\ldots$ & $1 / C_{n-1, n}$ & 1 \\
\hline
\end{tabular}

$O P V C=\left(W O c_{K P I 1}, W O C_{K P I 2}, \ldots W O c_{K P I n-1}, W O c_{K P I n}\right)$

$$
W O c=\sum_{i=1}^{n} W O c \mathrm{kpi}_{\mathrm{i}}
$$

OPVC should be normalised so that $W c O=1.0$

$$
O P C=O P V C X A O P V C^{T}
$$

$$
\begin{gathered}
O P V=\left(W O P_{C 1}, W O P_{C 2}, \ldots W O P_{C m-1}, W O P_{C m}\right) \\
A O P V=\left(A O P_{C 1}, A O P_{C 2}, \ldots A O P_{C m-1}, A O P_{C m}\right) \\
O P=O P V X A O P V^{T}(6) \\
\text { where } O P=\sum_{i=1}^{m} W O P_{C i} \mathrm{x} A O P_{C i} \\
S P V=\left(W S P_{C 1}, W S P_{C 2}, \ldots W S P_{C m-1}, W S P_{C m}\right) \\
A S P V=\left(A S P_{C 1}, A S P_{C 2}, \ldots A S P_{C m-1}, A S P_{C m}\right) \\
S P=S P V X A S P V^{T}(9) \\
\text { where } S P=\sum_{i=1}^{m} W S P_{C i} \mathrm{x} A S P_{C i} \\
R V=\left(W R_{C 1}, W R_{C 2}, \ldots W R_{C m-1}, W R_{C m}\right) \\
\mathrm{A} R V=\left(A R_{C 1}, A R_{C 2}, \ldots A R_{C m-1}, A R_{C m}\right) \\
R=R V X A R V^{T}(12) \\
w h e r e S=\sum_{i=1}^{m} W R_{C i} \times \mathrm{AR}_{C i}
\end{gathered}
$$

If the system has ' $m$ ' components contributing to the overall operational performance, the same approach is used to calculate the operational performance of each component and to assess the level of importance of these components to the overall system performance. The latter requires to develop a ' $\mathrm{m}$ x m' Priority Matrix to obtain the Operational Performance Vector for the system (OPV), equation (4), which has ' $\mathrm{m}$ ' components. The Actual Operational Performance Vector for the system (AOPV), equation (5), contains the actual performance for each component in the system. The overall system operational performance (OP) is obtained from the product of OPV and AOPV transposed, shown in equation (6).

Once again the same approach is used to assess the contribution of each component in the system to safety and resilience. The method assumes that safety and resilience are properties of the system provided by design that can be assessed by safety and resilience KPIs, respectively KSs and KRs, through agreed methods. Starting with safety, a Priority Matrix is constructed in the same way to obtain the Safety Performance Vector (SPV), equation (7). The Actual Safety Performance Vector (ASPV), equation (8), is obtained by assessing how well each KS is being met. The overall safety performance (SP) is the product of SPV and ASPV transposed, as shown in equation 9. 
Resilient systems can be achieved by capabilities within system or as an emergent property through collaboration with other systems. The first is achieved by design provided by redundant or backup components that second is achieved by emergent properties of SoS and will be discussed in the next section. System resilience can be assessed using the same methods as for performance and safety, as shown by equations (10) to (12).

$S I V=(W P, W S, W R)(13)$

$$
\begin{gathered}
A S I V=(P, S, R)(14) \\
S I=S I V X A S I V^{T}(15) ; \\
\text { where: } S I=W P_{X} P+W S_{X} S+W R \times R
\end{gathered}
$$

Table 4. Assessment Criteria Priority Matrix

Finally, it is also need to assess the level of importance of each of the three assessment criterion of operational performance, safety and resilience, to obtain the overall system integrity and the same approach is used once again, now in the form of a $3 \mathrm{x}$ 3 Priority Matrix (Table 4) shown in equations 13, 14

\begin{tabular}{|l|c|c|c|}
\cline { 2 - 4 } \multicolumn{1}{c|}{} & $\mathrm{O}$ & $\mathrm{S}$ & $\mathrm{R}$ \\
\hline Operational (O) & 1 & $\mathrm{PS}$ & $\mathrm{PR}$ \\
\hline Safety (S) & $\mathrm{SP}=1 / \mathrm{PS}$ & 1 & $\mathrm{SR}$ \\
\hline Resilience (R) & $\mathrm{RP}=1 / \mathrm{PR}$ & $\mathrm{RS}=1 / \mathrm{SR}$ & 1 \\
\hline
\end{tabular}
and 15. Equation (13) shows the System Integrity Vector (SIV) and equation (15) shows the overall System Integrity (SI) as the multiplication of SIV and the transposed Actual System Integrity Vector (ASIV), equation 14, which contains the actual system operational performance $(\mathrm{O})$, safety $(\mathrm{S})$ and resilience $(\mathrm{R})$.

It is reasonable to assume that safety would have a higher level of importance over operational performance and resilience. Operational performance could be considered a little higher than resilience because resilience is not expected to be activated frequently and some level of operational performance degradation may be acceptable. However, the weight of operational performance, safety and resilience should vary from system to system and should be estimated accordingly.

\section{ASSESSING SYSTEM OF SYSTEMS INTEGRITY: AN EXAMPLE}

System of Systems (SoS) comprise of many constituent systems that collaborate with each other in ways that the performance, safety and reliance of one constituent system may interfere with the performance, safety and reliance of other constituent systems, there system integrity (SI) and the overall SI for the SoS. The SoS SI model is represented in Figure 2.

AHP can be applied to systems with feedback loops where individual components and assessment criteria influence each other (Saaty 1994). AHP has been used in many complex systems applications including the investigation of emergent properties of SoS (DiMario, Boardman et al. 2009), the decision-making process to develop sustainable infrastructure (Diaz-Sarachaga, Jato-Espino et al. 2017) and to model a software-intensive acquisition for a naval helicopter (Peculis, Rogers et al. 2007). The method applying the SoS SI model using AHP extends what was presented in section 2 and will be presented as a hypothetical urban transport example.

Urban transport is a system of systems often comprising multi-mode forms of transport, a shared ticketing system, roads, parking and tolls. The example used to apply the System Integrity Assessment method to a SoS is a hypothetical simplified urban transport system comprising

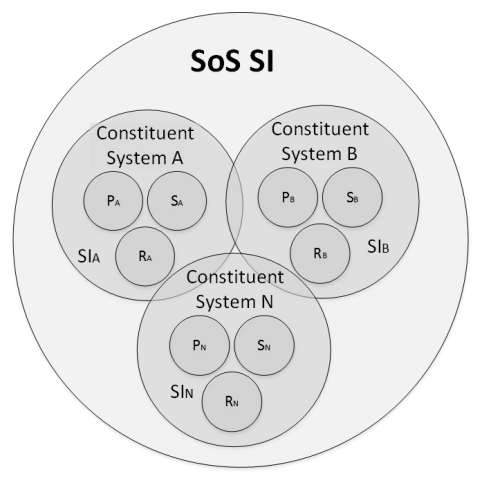

Figure 2. SoS SI Model

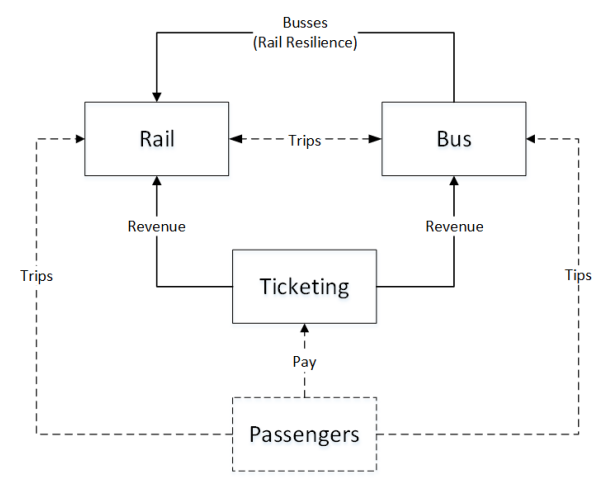

Figure 3. Hypothetical Urban Transport SoS of a network of trains and buses sharing a common ticketing system. Each of the constituent system (Rail, Bus and Ticketing) is independently managed and operated, and collaborates with the other systems to achieve the overall urban transport service, as shown in Figure 3. The three constituent systems work together to achieve three objectives of performance $(\mathrm{P})$, safety $(\mathrm{S})$ and resilience $(\mathrm{R})$ which are not equally shared by the constituent systems. Safety is very important for trains and buses but not as important for the ticketing system because the latter does not present the same 
level of safety risks as the other two. Performance also depends of different kinds of collaboration between the constituent systems. The flow of passengers between trains and buses and through the ticketing system is important for meeting the revenue KPI. Without Ticketing neither the Rail or Bus systems would be able to collect revenue and without passengers from train and buses the Ticketing system would not be able to collect its share for the ticketing service. Passengers, trips and payments, represented by dotted lines, are not directly managed by the constituent systems but are may be reflected by the various KPIs.

To assess the system integrity of this urban transport SoS the method proposed in section 2 is extended with four additional steps as indicated in Table 5.

Table 5. Extended steps for assessing the SoS SI

\begin{tabular}{|l|l|}
\hline Step 7 & $\begin{array}{l}\text { Estimate the level of importance of each constituent system for each of the three criteria of performance, safety } \\
\text { and resilience using AHP supermatrix approach. }\end{array}$ \\
\hline Step 8 & $\begin{array}{l}\text { Estimate the relevance of each of the three criteria relevant for each of the three constituent systems is assessed } \\
\text { using AHP supermatrix approach. }\end{array}$ \\
\hline Step 9 & $\begin{array}{l}\text { Calculate the relative weight of each constituent systems and each individual criterion by stabilising the AHP } \\
\text { supermatrix. }\end{array}$ \\
\hline Step 10 & $\begin{array}{l}\text { Calculate the SI for the SoS using the individual SI for each constituent system applying steps } 1 \text { to } 6 \text { and the } \\
\text { weights calculated in step 9. }\end{array}$ \\
\hline
\end{tabular}

For the example here presented the Bus system collaborates with the Rail system to provide resilience. Buses can replace trains for a particular section of the Rail line when it is not operating due to failure or maintenance. The inverse, however, is not possible because in practice trains are unlikely to be able to replace buses in a significant portion of their routes.

\section{Steps 7 and 8}

To assess the system integrity of this urban transport SoS it is needed to weight the contribution of each constituent systems to the overall SI and also weight the three criteria of performance, safety and resilience in the context of the SoS as a whole.

The bottom left part of the supermatrix in Table 6 answers the question 'what is the level of importance of each constituent system for each of the three criteria of performance, safety and resilience?'. The top right part of the supermatrix answers the question of 'what of the three criteria is more relevant for each of the three constituent systems?'. The weights placed in the supermatrix reflect the characteristics of the urban transport SoS and could have been estimated using Priority Matrices as discussed in section 3. Here, however, the weights were estimated directly for simplicity and hypothetical nature of this example.

\section{Step 9}

The sum of the weights of each column of the supermatrix is equal to 1.0 which is characteristic of 'stochastic supermatrix' which can be stabilised by raising it to power, i.e. multiplying the matrix by itself several times, until all the columns have the same values for each block, as shown in Table 7.

The three weights shown at the bottom left of the matrix correspond to WP, Ws and WRs of equation (13) and are used to calculate the SI for each constituent systems as per equations (14) and (15). The three weights at the top right of the matrix are the relative weights for the SI of each constituent system and form the Urban
Table 7. Hypothetical Urban Transport SoS stabilised Supermatrix

\begin{tabular}{|l|c|c|c|c|c|c|}
\cline { 2 - 7 } \multicolumn{1}{c|}{} & $\mathrm{R}$ & $\mathrm{B}$ & $\mathrm{T}$ & $\mathrm{P}$ & $\mathrm{S}$ & $\mathrm{Rs}$ \\
\hline Rail (R) & 0 & 0 & 0 & 0.5457 & 0.5457 & 0.5457 \\
\hline Bus (B) & 0 & 0 & 0 & 0.3114 & 0.3114 & 0.3114 \\
\hline Ticketing (T) & 0 & 0 & 0 & 0.1429 & 0.1429 & 0.1429 \\
\hline Performance (P) & 0.4286 & 0.4286 & 0.4286 & 0 & 0 & 0 \\
\hline Safety (S) & 0.4571 & 0.4571 & 0.4571 & 0 & 0 & 0 \\
\hline Resilience (Rs) & 0.1143 & 0.1143 & 0.1143 & 0 & 0 & 0 \\
\hline
\end{tabular}

$$
U T S I V=(W R, W B, W T)(16)
$$

$$
\text { AUTSI } V=(A S I R, A S I B, A S I T)(17)
$$$$
U T S I=U_{T S I V} \operatorname{AUTSIV}^{T}(18)
$$

where: $U T S I=W R \times A S I R+W B \times A S I B+W T \times A S I T$ 
Peculis and Shirvani, Assessing Infrastructure System of Systems Integrity

Transport System Integrity vector (UTSIV) as per equation (16) and the Actual Urban Transport System Integrity Vector (AUTSIV) is shown in equation (17).

\section{Step 10}

Finally, the system integrity of the urban transport SoS is calculated by multiplying UTSIV by the Actual Urban Transport System Integrity Vector (AUTSIV) transposed, as per equation (18), where ASIR, ASIB and ASIT are respectively the actual values for SI for Rail, Bus and the Ticketing constituent systems calculated using the method presented in section 2 .

\section{CONCLUSIONS}

This paper proposed a model and a method to assess system integrity for systems and system of systems using AHP. The method relies on the human ability of performing pairwise comparison and in the capability of engineers and managers to define and assess key indicators of performance, safety and resilience for the systems they are responsible for. The method also assumes that engineers and managers would be able to agree on KPIs, their respective methods of assessment and the on the relative weights for each of their assessment criterion. The proposed method allows infrastructure professionals to identify KPIs and components in the system or SoS that have higher influence on SI which in turn should have higher priority for improvements, issues identification and resolution.

The authors acknowledge that uncertainty and lack of confidence can be present in the process of developing Priority Matrices and relative weights between the elements in the system. Techniques like fuzzy hierarchical analysis (Buckley 1985) can be used to address uncertainty in the process of assessing system integrity and will be subject of future work. The proposed model and method are yet to be tested in practice and its application will be important for future work addressing real systems and SoS with more components, constituent systems, interdependencies and assessment criteria set. In the meantime the authors hope that this paper will be able to motivate infrastructure managers and decision-makers to consider the application of this method into their own real systems and SoS. It is of great importance that these professionals reflect upon the definition of system integrity in the context of their specific systems, which should lead to the identification of system components, constituent systems, assessment criteria and weight of relevance, influence and importance. The method here proposed should provide guidance for a good start.

\section{REFERENCES}

American Heritage Dictionary (2017). "American Heritage ${ }^{\circledR}$ Dictionary of the English Language." Retrieved 11 July 2017, from http://www.thefreedictionary.com/integrity.

Buckley, J. J. (1985). "Fuzzy hierarchical analysis." Fuzzy sets and systems 17(3): 233-247.

Business Dictionary (2017). "Business dictionary." Retrieved June 17: 2017.

Diaz-Sarachaga, J. M., et al. (2017). "Methodology for the development of a new Sustainable Infrastructure Rating System for Developing Countries (SIRSDEC)." Environmental Science \& Policy 69: 65-72.

DiMario, M. J., et al. (2009). "System of systems collaborative formation." IEEE Systems Journal 3(3): 360368.

Kapurch, S. J. (2010). NASA systems engineering handbook, Diane Publishing

Madni, A. M. and M. Sievers (2014). "Systems integration: Key perspectives, experiences, and challenges." Systems Engineering 17(1): 37-51.

Neches, R. and A. M. Madni (2013). "Towards affordably adaptable and effective systems." Systems Engineering 16(2): 224-234.

Nielsen, C. B., et al. (2015). "Systems of systems engineering: basic concepts, model-based techniques, and research directions." ACM Computing Surveys (CSUR) 48(2): 18.

Peculis, R., et al. (2007). A Task Model of Software Intensive Acquisitions: An Integrated Tactical Avionics System Case Study. Twelfth Australian Aeronautical Conference, Engineers Australia.

Pyster, A., et al. (2017). "Guide to the systems engineering body of knowledge (SEBoK) v. 1.8." Guide to the Systems Engineering Body of Knowledge (SEBoK).

Saaty, T. L. (1994). Fundamentals of Decision Making and Prority Theory with the Analytic Hierarchy Process, RWS Publications. 